\title{
Three-Dimensional Characterization of Polycrystalline Materials by Combination of X-ray Diffraction and X-ray Imaging Techniques
}

\author{
E.M. Lauridsen, ${ }^{*}$ W. Ludwig, ${ }^{* *}$ S.O. Poulsen, ${ }^{*}$ S. Rolland du Roscoat,** P. Reischig, ${ }^{* *}$ A. \\ King, ${ }^{* * *}$ A. Lyckegaard,* and R.W. Fonda**** \\ * Risø National Laboratory for Sustainable Energy, Technical University of Denmark, P.O. Box 49, \\ 4000 Roskilde, Denmark \\ ** European Synchrotron Radiation Facility, BP220, 38043 Grenoble, France \\ *** School of Materials, University of Manchester, Manchester, M13 9PL, UK \\ **** Naval Research Laboratory, Washington, DC, 20375
}

Three-dimensional characterization has proven it self highly valuable for the understanding of complex microstructures in material science. In several cases such three-dimensional characterizations has demonstrated that previous conclusions based solely on two-dimensional investigations are incomplete or even incorrect. As a direct consequence there has been increasing interest in development and improvement of three-dimensional characterization tools for use within the materials science field (for an overview see e.g. [1][2]).

Synchrotron based 3D imaging techniques constitute a family of 3D characterization tools which has the attractive property that they are non-destructive and thereby allow for dynamic studies. Within the last decade a whole range of new 3D synchrotron techniques has emerged which compliments the capabilities of the well-known conventional absorption tomography technique.

In this presentation we will provide an overview of the existing 3D synchrotron techniques, their advantages and limitations. Special focus will be on how combinations of diffraction techniques and imaging techniques can be used to obtain more complete 3D characterization of polycrystalline materials. This will be illustrated with examples within materials science. [3]

References

[1] K. Thornton \& H.F. Poulsen, MRS Bullitin Vol. 33(6) "Three-Dimensional Materials Science” (2008), 587-629

[2] G. Spanos, Scripta Mater. Vol. 55(1) Viewpoint set no. 41 “3D Characterization and Analysis of Materials" (2006) 1-114

[3] The authors thank the European Synchrotron Radiation Facility for provision of beam time. E.M.L., W.L., S.P. and A.L. acknowledge the Danish National Research Foundation and the Danish Natural Science Research Council (via DANSCATT) for supporting the Center for Fundamental Research: Metal Structures in 4D, within which part of this work was performed and E.M.L. and R.W.F. furthermore acknowledge the support jointly sponsored by the Office of Naval Research and DARPA as part of the Dynamic 3-D Digital Structure Program (Grant numbers N00015-05-1-0510 \& N00014-08-WX20726). A.K. acknowledges funding received from the Engineering and Physical Sciences Research Council. 


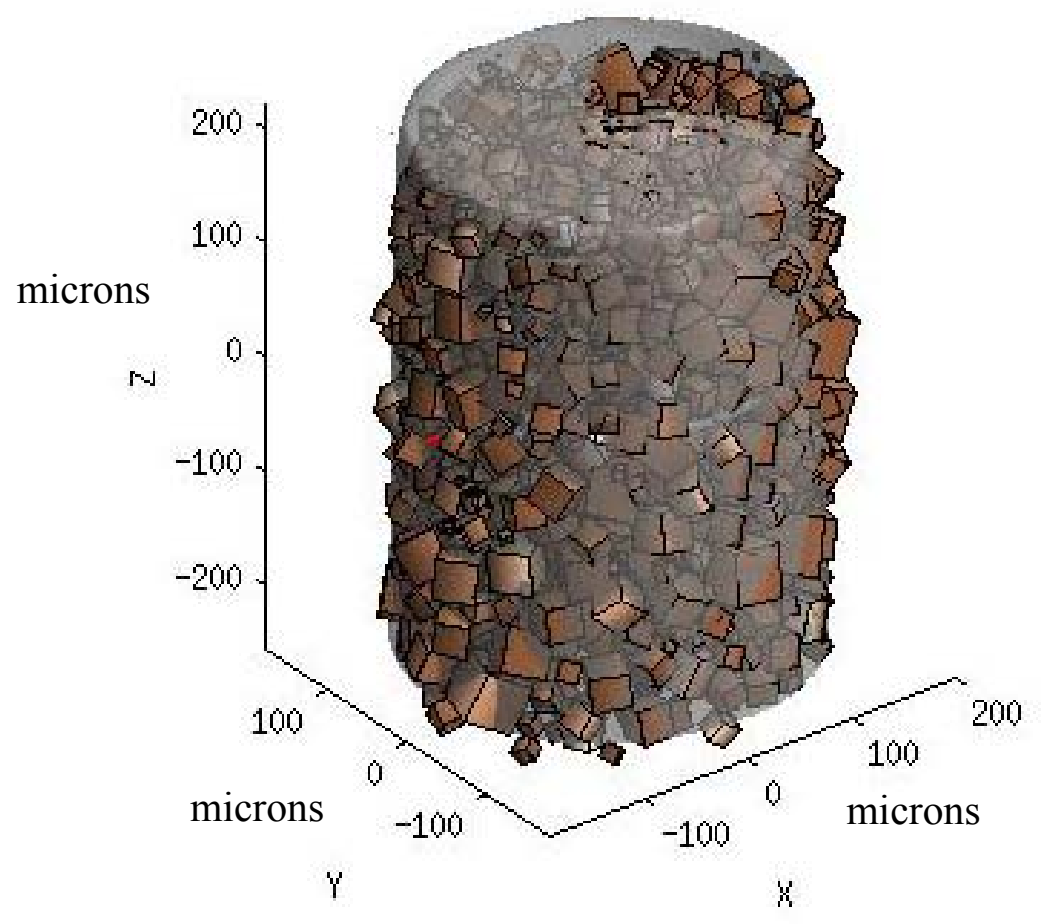

FIG. 1. Example of visualization of the position and crystallographic orientation of $\sim 1000$ grains in a Timet $\beta 21 \mathrm{~s}$ titanium alloy. The sizes of the cubes are proportional to the grain sizes. The data is acquired using diffraction contrast tomography at the ID19 beam line at the European Synchrotron Radiation Facility, Grenoble, France.

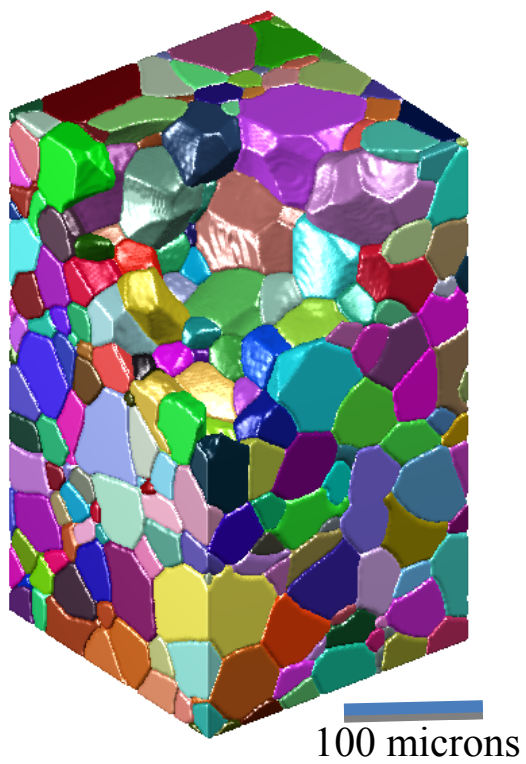

FIG. 2. Example of visualization of the grain structure of a Timet $\beta 21 \mathrm{~s}$ titanium alloy (the colors in the figure are random). The data is acquired using phase contract tomography at the ID19 beam line at the European Synchrotron Radiation Facility, Grenoble, France. 